\title{
Global neuroinflammation patterns in experimental neonatal hydrocephalus
}

\author{
James P McAllister II*, Jennifer Forsyth and Kelley E Deren
}

\author{
Address: Department of Neurosurgery, Primary Children's Medical Center, Salt Lake City, Utah, USA \\ Email: James P McAllister* - pat.mcallister@hsc.utah.edu \\ * Corresponding author
}

from 53rd Annual Meeting of the Society for Research into Hydrocephalus and Spina Bifida Belfast, UK. 24-27 June 2009

Published: 27 November 2009

Cerebrospinal Fluid Research 2009, 6(Suppl 2):SI2 doi:I0.II86/1743-8454-6-S2-SI 2

This abstract is available from: http://www.cerebrospinalfluidresearch.com/content/6/S2/SI2

(c) 2009 McAllister et al; licensee BioMed Central Ltd.

\section{Background}

Hydrocephalus causes reactive astrocytosis and microgliosis throughout the brain but the global response of proinflammatory cytokines is not known. Thus we sought to characterize common inflammatory markers in a neonatal model of obstructive hydrocephalus. Our main hypothesis was that neuroinflammation would progress in neonatal hydrocephalus proportional to ventriculomegaly. In addition, the long-standing uncertainty about the possible global inflammatory effects of kaolin could also be addressed.

\section{Materials and methods}

Intraventricular (obstructive) hydrocephalus was induced in one-day old Sprague-Dawley rats by intracisternal injections of $25 \%$ kaolin ( $n=6-7$ per tissue sample); agedmatched controls received similar injections of saline $(\mathrm{n}=$ 3-6 per tissue sample). MRI was employed to characterize ventriculomegaly, and animals were sacrificed on postnatal day 21. Tissue from the frontal cortex, parietal cortex, hippocampus, midbrain (tectum and tegmentum), and medulla was analyzed. Quantitative real time reverse transcriptase polymerase chain reaction (qRT-PCR) was performed to determine changes in mRNA expression of interleukin 6 (IL-6), tumor necrosis factor alpha (TNFalpha), glial fibrillary acidic protein (GFAP), and major histocompatibility complex class II (MHC-II). Protein expression was also examined in these animals by Western blotting. Correlative immunocytochemistry was also performed.

\section{Results}

All portions of the cerebral ventricles expanded, especially in the lateral ventricles where ventriculomegaly was severe and in the cerebral aqueduct where the posterior recess was separated from the overlying subarachnoid space (which also expanded) by only a thin membrane. Both the frontal and parietal cortices exhibited significant $(p<0.05)$ increases in IL-6, TNF-alpha, GFAP and MHC-II. Likewise, the midbrain exhibited statistically significant increases in IL-6, TNF-alpha, GFAP and MHC-II. In contrast, the medulla, which was the only region adjacent to kaolin deposits, showed no change in IL-6, TNF-alpha and GFAP, and only a modest non-significant increase in MHC-II.

\section{Conclusion}

These results suggest that severe hydrocephalus causes inflammatory changes in the cerebral cortex and midbrain of the developing brain which are probably a direct consequence of ventriculomegaly. Data from the medulla suggest that kaolin itself is not directly involved in a widespread inflammatory response. Characterizing the time course and association with ventricular volume will provide baseline data for future studies on pharmacological interventions. In this regard, our previous findings of significant decreases in the number of reactive astrocytes and microglia with minocycline treatment suggest that this agent may also reduce neuroinflammation in hydrocephalus. 